\section{An unbiased catch}

More and more, genome-wide linkage scans for complex trait loci, such as those for hypertension or asthma, rely on pooled data because individual scans do not generate enough data to accurately point to their location. But pooled data has to be analysed carefully to avoid any bias that could result in a false linkage prediction. Even the flexible and popular Fisher's method, which offers a way of obtaining a linkage probability by combining evidence from many different statistical tests, is not free of bias because it tends to underplay the importance of negative evidence against linkage. In the American Journal of Human Genetics, Michael Province shows that this bias can be overcome with a simple correction.

The LOD (logarithm of the odds) score is a statistical estimate of linkage. Under parametric, or model-based, analyses, the LOD score can be transformed into a $P$-value - the probability that the locus or loci are linked to a trait - because the LOD calculation itself is based on probability: $\mathrm{LOD}=0$ corresponds to $P=0.5$, and $P>0.5$ and $P<0.5$ correspond to negative and positive LOD scores, respectively. Several tests of linkage can then be combined by using Fisher's procedure, which allows the probability values from independent statistical tests to be pooled, to yield an overall probability of linkage. However, in non-parametric, or model-free, analyses, the direct correspondence between a LOD score and the probability is lost because all evidence against linkage is truncated to a LOD score of zero. If we interpret these LOD scores as $P=0.5$, the overall test of linkage using Fisher's procedure is liberal (that is, the probability of linkage is overestimated), and if we interpret LOD $=0$ as $P=1$, the test becomes conservative.

Michael Province shows both analytically and by using computer simulations that to avoid almost all the bias from the combining probabilities procedure LOD $=0$ scores should be interpreted as $P \approx 0.72$. Although a much more complex adjustment is necessary to remove all bias, this simple modification of Fisher's method is sufficient for studies in which fewer than 100 samples have been pooled. This simple correction should make it easier to combine the results of different genome-fishing trips for complex traits.

Magdalena Skipper

(2) References and links

ORIGINAL RESEARCH PAPER Province, M. A. The significance of not finding a gene. Am. J. Hum. Genet. 69, 660-663 (2001)

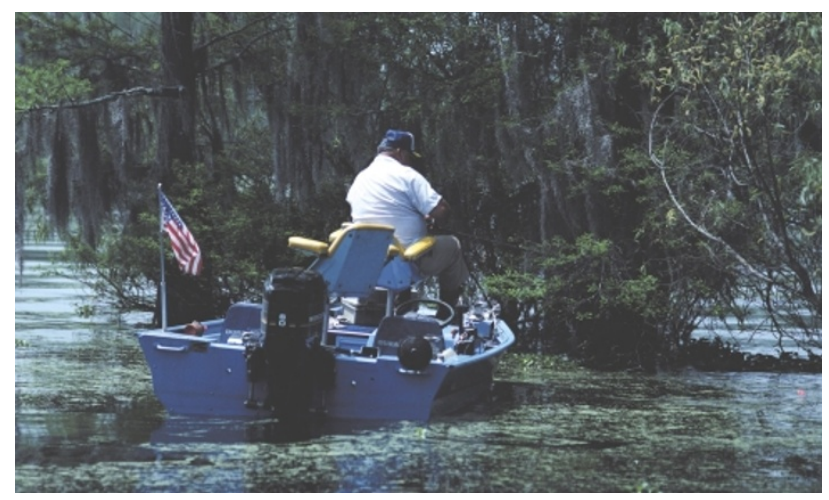

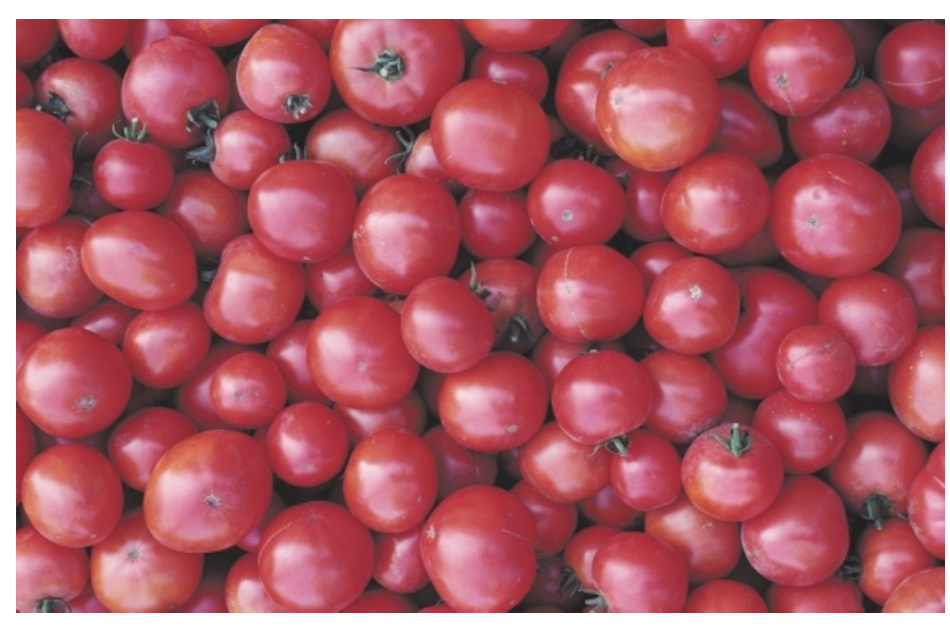

TECHNOLOGY

\section{Fruitful}

\section{transgenesis}

Genetically modified (GM) tomatoes have been around since the mid1990s, when scientists created transgenic tomatoes with a prolonged ripening period. In the September issue of Nature Biotechnology, Ruf et al. describe the first successful application of a new transformation method plastid transgenesis - in tomato. This technique, in which foreign genes are targeted to a cytoplasmic genome, has advantages over the more conventional, nuclear transformation; notably, high expression of the transgene in the fruit offers the promise of using tomatoes to produce biopharmaceuticals and to engineer fruit with a higher nutrient content.

Plastids, such as chloroplasts, are cytoplasmic organelles with small, highly polyploid genomes that, unlike nuclear DNA, are free of higher-order chromatin and epigenetic modification — allowing efficient homologous recombination and high levels of gene expression to occur. Importantly for the environmental impact of GM crops, plastids - like all cytoplasmic organelles - are almost always inherited maternally, thereby eliminating the risk that transgenes will spread through pollen to other plants.

As the aim of the experiment was to induce strong transgene expression in edible plant parts, the authors used a ribosomal RNA promoter Prrnknown to be active in fruit. Transgenic constructs were delivered in culture to plant tissues by gold particle bombardment and integration of the transgenic construct into the plastid genome by homologous recombination was ensured by flanking the foreign genes with plastid-derived 'targeting' sequences. Transformants were then selected on the basis of the antibiotic resistance conferred by the construct and by PCR.

The polyploid nature of the plastid genome has its disadvantages. Because a single cell can contain as many as 10,000 genomes, it is difficult to select pure transgenic lines. The success of plastid transgenesis therefore relies on having a robust method to select transformants: Ruf et al. targeted small leaf pieces for transformation, and carried out all their selection procedures under very low light conditions and over a long period of time. Lack of mosaicism in the tissues was then confirmed by restriction fragment length polymorphism analysis, after which the transformed tissues were used to regenerate a whole plant.

Using this protocol, the authors found that the transgene was expressed throughout the plant, including the fruit, where the transgene product made up $0.5 \%$ of the total soluble protein content, making it the first successful transgene to be expressed to high levels in the edible parts of a plant.

The technique needs to be optimized before it is commercially useful - shorter selection times are desirable, for example. On the basis of expression studies in tobacco, the authors suggest that the levels of transgene expression in the fruit can reach up to $20 \%$ of total soluble protein content. These results open up many appetizing opportunities for biotechnologists, who are always eager to find new ways in which to produce and deliver vaccines, drugs and nutrients.

Magdalena Skipper

6) References and links ORIGINAL RESEARCH PAPER Ruf, S. et al. Stable genetic transformation of tomato plastids Stable genetic transformation of tomato plastids
and expression of a foreign protein in fruit. Nature Biotechnol. 19, 870-875 (2001)

FURTHER READING Maliga, P. Plastid engineering bears fruit. Nature Biotechnol. 19, 826-827 (2001) WEB SITE

Ralph Bock's lab: http://www.biologie.unifreiburg.de/data/bock/resreprt.htm 